\title{
Long-Term Quality of Life after Decompressive Craniectomy in Severe Traumatic Brain Injury and Stroke
}

\author{
Vicino $A^{1}$, Vuadens $P^{2}$, Léger $B^{3}$ and Benaim $C^{3,4 *}$ \\ ${ }^{1}$ Neurology Service, Department of Clinical \\ Neurosciences, Lausanne University Hospital and \\ University of Lausanne, Lausanne, Switzerland \\ ${ }^{2}$ Department of Neurorehabilitation, Clinique Romande \\ de Réadaptation, Sion, Switzerland \\ ${ }^{3}$ Department of Medical Research, Clinique Romande de \\ Réadaptation, Sion, Switzerland \\ ${ }^{4}$ Department of Physical Medicine and Rehabilitaion, \\ Lausanne University Hospital and University of \\ Lausanne, Lausanne, Switzerland \\ *Corresponding author: Charles Benaim, Physical \\ Medicine and Rehabilitation, Centre Hospitalier \\ Universitaire Vaudois (CHUV), Avenue Pierre Decker 4, \\ 1011 Lausanne, Switzerland
}

Received: J une 21, 2021; Accepted: August 04, 2021; Published: August 11, 2021

\begin{abstract}
Purpose: Decompressive Craniectomy (DC) can rapidly reduce intracranial pressure and save lives in the acute phase of severe Traumatic Brain Injury (TBI) or stroke, but little is known about the long-term outcome after DC. We evaluated Quality of Life (QoL) a few years after DC for severe TBI/stroke.
\end{abstract}

Methods: The following data were collected for stroke/TBI patients hospitalized for neurorehabilitation after DC: 1) at discharge, motor and cognitive sub-scores of the Functional Independence Measure (motor-FIM (score 13-91) and cognitive-FIM (score 5-35)) and 2) more than 4 years after discharge, the QOLIBRI health-related QoL (HR-QoL) score $(0-100 ;<60$ representing low or impaired QoL) and the return to work (RTW: 0\%, partial, 100\%).

Results: We included 88 patients ( 66 males, median age 38 (interquartile range 26.3-51.0), 65 with TBI/23 stroke); 46 responded to the HR-QoL questionnaire. Responders and non-responders had similar characteristics (age, sex, functional levels upon discharge). Median motor-FIM and cognitiveFIM scores were $85 / 91$ and $27 / 35$, with no significant difference between TBI and stroke patients. Long-term QoL was borderline low for TBI patients and within normal values for stroke patients (score 58.0 (42.0-69.0) vs. 67.0 (54.081.5), $p=0.052$ ). RTW was comparable between the groups ( $62 \%$ full time).

Conclusion: We already knew that DC can save the lives of TBI or stroke patients in the acute phase and this study suggests that their long-term quality of life is generally quite acceptable.

Keywords: TBI; Stroke; Decompressive carniectomy; Quality of life

\section{Abbreviations}

DC: Decompressive Craniectomy; FIM: Functional Independence Measure; HR-QoL: Health-Related Quality of Life; QoL: Quality of Life; RTW: Return to Work; TBI: Traumatic Brain Injury

\section{Background and Purpose}

Stroke and Traumatic Brain Injury (TBI) are among the leading causes of death and disability in developed countries. In Switzerland, they have an incidence of approximately 240/100,000 and 170/100,000 (8.2/100,000 considered severe) [1-3], and represent two of the leading causes of death, with mortality at $14.4 \%$ for stroke and $20 \%$ to $30 \%$ for TBI in the first year and $50 \%$ and $35 \%$ in the following 5 years. These two conditions also induce major chronic disability in the population, with a significant impact on quality of life (QoL) $[4,5]$.

In severe stroke or TBI, high Intracranial Pressure (ICP) can be life-threatening. Decompressive Craniectomy (DC) is widely used to avoid refractory intracranial hypertension, one of the most common causes of death after stroke or TBI. As described by the Cochrane Collaboration in the 2016 Brain Trauma Foundation guidelines, DC is recommended as second-line treatment for TBI [6]. However, this latest version of these guidelines does not recommend DC to improve outcomes, although it acknowledges that the procedure is useful for rapidly reducing ICP and minimizing days spent in the intensive care unit. DC is recommended in Switzerland as Class I/
Level A management for middle cerebral artery stroke with altered consciousness and mass effect [7] and, according to the classification of the European Federation of neurological societies guidelines for therapeutic interventions, as Class III/Level C management for cerebellar infarction [8]. The exact proportion of patients requiring craniectomy after TBI or malignant ischemic stroke remains unknown because no national incidence data are available. In a Swiss cohort of 101 patients with TBI, 60 survived the accident and 9 (15\%) underwent DC [1]. Despite immediate benefits in reduced ICP and mortality, the effect of DC on disability is still controversial.

We collected information on long-term ( $>4$ years) health-related QoL (HR-QoL) and occupational outcomes in patients with stroke or TBI who underwent DC and were hospitalized in our rehabilitation clinic. Another objective was to compare HR-QoL and RTW in the two groups.

\section{Methods}

\section{Study design and setting}

This was a monocentric retrospective cross-sectional study conducted in the Clinique Romande de Réadaptation, a nationalreference rehabilitation center in Switzerland that receives patients who underwent DC after stroke or TBI.

\section{Participants}

Eligible patients were those admitted in our neurorehabilitation
Phys Med Rehabil Int - Volume 8 Issue 3 - 2021

ISSN : 2471-0377 | www.austinpublishing group.com

Benaim et al. (C) All rights are reserved
Citation: Vicino A, Vuadens P, Léger B and Benaim C. Long-Term Quality of Life after Decompressive Craniectomy in Severe Traumatic Brain Injury and Stroke. Phys Med Rehabil Int. 2021; 8(3): 1186. 
unit between 2003 and 2012 and had undergone DC for malignant stroke or TBI. Exclusion criteria was DC for another intracranial pathology (cancer, subarachnoid hemorrhage etc.). The study was approved by the state ethics committee (CER-VD, 2017-00458). All participants or their representatives gave their informed consent before the start of the enrolment.

\section{Variables/data measurement}

At discharge, the motor and cognitive sub-scores of the Functional Independence Measure (motor-FIM and cognitive-FIM) were used to characterize patients' functional abilities [9]. Between 4 to 14 years after discharge from the clinic, patients were 1) invited by mail to complete the QOLIBRI questionnaire (HR-QoL) [10], a 37-item scale that assesses both physical- and psychological-related life satisfaction, resulting in an overall satisfaction score (0-100, 100 being the best possible QoL; a score $<60$ represents low or impaired QoL [11]) and 2) asked about their return to work (RTW), rated as $0 \%$, partial or $100 \%$ if they returned to the occupation held before the stroke or TBI. Two reminders were sent after 2 and 4 weeks in case of non-response. Patients who still did not answer were contacted by phone after another 2 to 4 weeks.

\section{Study size}

Because of no previous data on the topic, we were unable to estimate a suitable number of participants needed for this study. Rather, we considered 40 to 50 participants as a reasonable sample size to estimate patient outcome after DC, given that this is a relatively rare surgery. To account for non-response, we set the total number of participants at 80 .

\section{Statistical methods}

Differences between groups (responders/non-responders, stroke/ TBI) were analyzed with the non-parametric Wilcoxon rank-sum test for quantitative variables and chi-square test for categorical variables. Data were analyzed with NCSS Number Cruncher Statistical System
(2013: Atlanta). $\mathrm{P}<0.05$ was considered statistically significant.

\section{Results}

\section{Participants (Figure 1)}

From the 95 consecutive patients hospitalized at the Clinic during the inclusion period after a DC, 88 met the inclusion criteria, but only 46 (52\%) completed the HR-QoL questionnaire and 45 (51\%) had data on RTW, despite the reminders. Responders and non-responders did not differ in age and sex. They progressed to similar functional levels upon discharge from the clinic (FIM (Median [Interquartile range]): 111.5 [86.8-120.0] vs. 109.0 [77.0-119.0], p=0.553). However, among the FIM items, "memory" was significantly higher in responders than non-responders (5.5 [4.8-6.3] vs. 5.0 [4.0-5.8], $\mathrm{p}=0.032)$.

\section{Descriptive and outcome data (Table 1)}

TBI patients $(n=65)$ were slightly younger and more often males than stroke patients $(n=23)$. Patients had left the clinic between 4.4 and 13.8 years earlier, with no difference between groups (8.1 [5.710.8] vs. 9.2 [6.9-11.6] years for TBI and stroke, $\mathrm{p}=0.24)$. Median HRQoL score was lower for TBI than stroke patients (58.0 [42.0-69.0] vs. 67.0 [54.0-81.5], $\mathrm{p}=0.052$ ). Most patients reported full-time RTW; the no-RTW proportion was lower but not significantly for TBI than stroke participants $(\mathrm{p}=0.192)$.

\section{Discussion}

To our knowledge, this is the first study investigating QoL and RTW outcomes after DC in Switzerland, with a comparison between two etiologies. Our cohort study of 88 stroke and TBI patients after DC showed that long-term QoL was within the normal range after stroke and was borderline low after TBI [11]. The median HR-QoL score was significantly lower after TBI than stroke, but the percentage of RTW was slightly higher after TBI (not significant). The overall functional status after hospital discharge was comparable between the two groups and could not explain the difference in QoL.

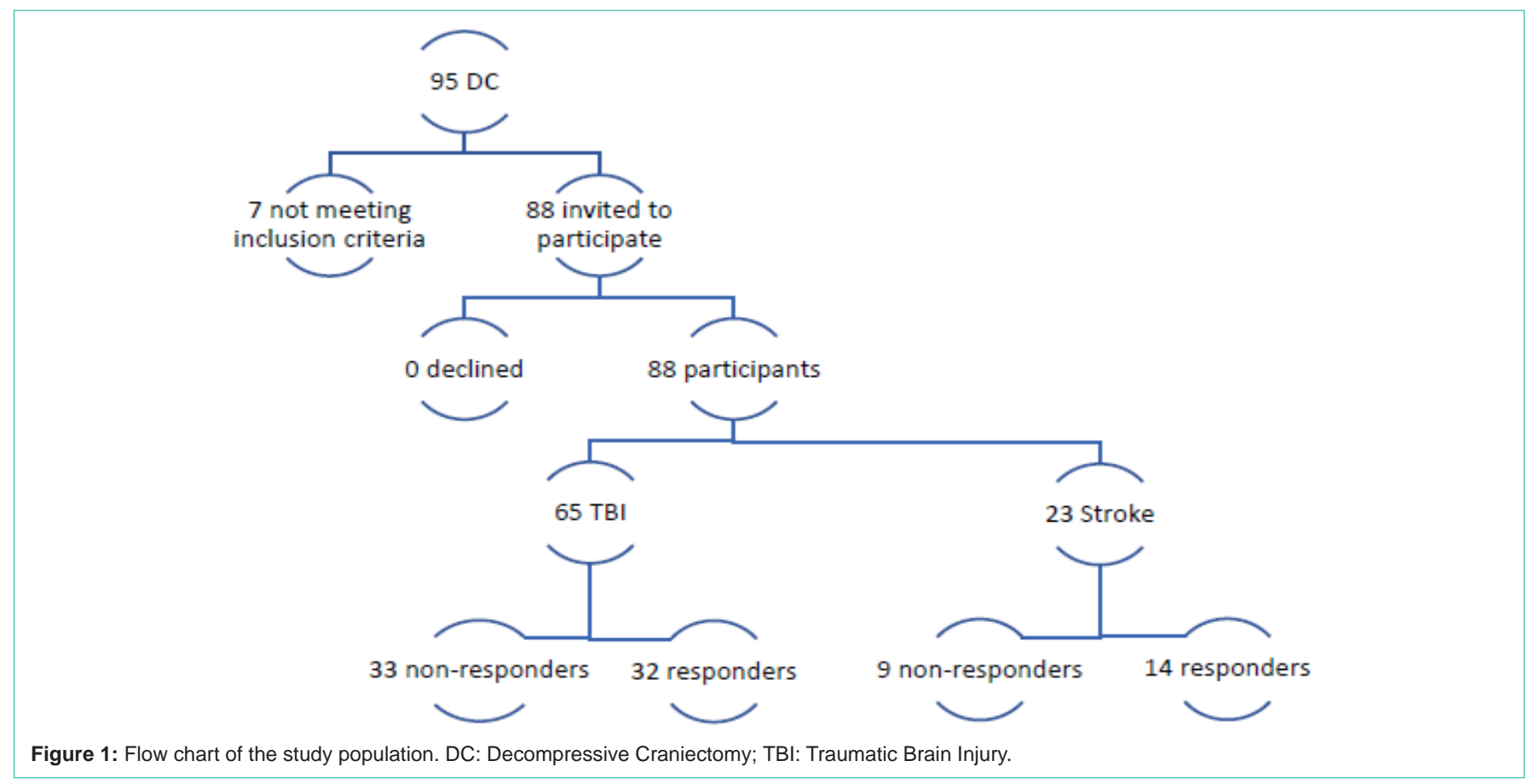


Table 1: Baseline characteristics of participants with Traumatic Brain Injury (TBI) or stroke and data after 4 years.

\begin{tabular}{|c|c|c|c|c|}
\hline Variable & Overall $(n=88)$ & TBI $(n=65)$ & Stroke $(n=23)$ & P-value \\
\hline Age & 38.0 [26.3-51.0] & 35.0 [29.0-43.0] & $46.0[36.0-55.0]$ & 0.093 \\
\hline $\operatorname{Sex}(M)$ & $66(75 \%)$ & $55(85 \%)$ & $11(48 \%)$ & 0 \\
\hline \multicolumn{5}{|l|}{ FIM } \\
\hline mot-FIM (13-91) (n=79) & 85 [77-91] & 88 [81-90] & 81 [75-91] & 0.352 \\
\hline cog-FIM (5-35) $(n=68)$ & 27 [22-31] & 27 [25-29] & 28 [22-31] & 0.87 \\
\hline total FIM (/126) $(n=80)$ & 111 [89-119] & 112 [104-118] & $104[92-117]$ & 0.504 \\
\hline Responders & $46(52 \%)$ & $32(49 \%)$ & $14(61 \%)$ & 0.337 \\
\hline HR-QoL $(/ 100)(n=44)$ & $62.5[45.8-73.0]$ & $58.0[42.0-69.0]$ & $67.0[54.0-81.5]$ & 0.052 \\
\hline \multicolumn{5}{|l|}{ RTW (n=45) } \\
\hline Full time & $28(62 \%)$ & $22(71 \%)$ & $6(43 \%)$ & \multirow{3}{*}{0.192} \\
\hline Part time & $9(20 \%)$ & $5(16 \%)$ & $4(29 \%)$ & \\
\hline No & $8(18 \%)$ & $4(13 \%)$ & $4(29 \%)$ & \\
\hline
\end{tabular}

Data are median [interquartile range] unless indicated. TBI: Traumatic Brain Injury; FIM: Functional Independence Measure; mot-FIM: FIM motor Subscore; cog-FIM: FIM Cognitive Subscore; HRQoL: Health-Related Quality of Life; RTW: Return to Work.

Median HR-QoL score seems to be lower in our TBI patients than in the QOLIBRI validation study patients (median 58.0 [IQR 42.0-69.0] vs. 64.58 \pm 18.24 ) probably because all of our patients had a malignant ICP. In the past few years, two multi-center randomized controlled studies helped to better define the outcome of DC. The DECRA study analyzed a cohort of 155 adults randomly assigned to bifrontal DC or standard medical care. DC decreased ICP and length of stay in the intensive care unit but was associated with less favorable outcomes than standard care [12]. However, QoL was not specifically measured. The RESCUE-icp study was a large multicenter randomized controlled trial including 408 TBI patients [13]. When compared to standard medical care, DC resulted in lower mortality rate and higher rates of vegetative state and severe disability but similar levels of moderate disability and good recovery after 6 months. Although long-term data collection was planned in this study (including QoL), those data were not published. In a recent cross-sectional German study, considering all levels of severity of TBI treated with or without DC, DC was associated with better HR-QoL up to 10 years after TBI (median HR-QoL score: 75 vs. 67) [14].

Previous studies described an average rate of RTW of $35.4 \%$ to $59.5 \%$ for stroke and $30 \%$ to $65 \%$ for TBI (lower for severe TBI), with an increase in this percentage over time [15]. RTW rates in our cohort were slightly better for stroke patients and were within the expected range for TBI patients.

Our study had several limitations, the main one being that just over half of the patients responded to the HR-QoL survey. Nonresponse by participants is a common problem in this kind of survey and is difficult to remedy. For example, the response rate in the Rauen et al. study was only $31 \%(135 / 439)$ [14]. In our study, responders and non-responders had the same demographic characteristics and had achieved the same overall functional level at discharge. This allows us to assume that the long-term evolution should not have been very different between the two groups of patients, and therefore minimizes the selection biase. Another limitation is that, although the study was conducted in a national reference center, we could not include a sufficient number of patients to assess the effect of co-variables on the outcome. Only a multicenter (international) study design could achieve this goal.

\section{Conclusion}

Not only can DC save patients' lives in the days following a severe TBI or stroke, but patients treated with this technique generally have a quite acceptable quality of life in the long term.

\section{Declaration}

Ethic approval and consent to participate: All procedures performed in this study were in accordance with the ethical standards of the state research committee and with the 1964 Helsinki declaration and its later amendments. The study was approved by the state ethics committee (CER-VD, 2017-00458). All participants or their representatives gave their informed consent before the start of the enrolment.

Authors' contributions: All authors substantially contributed to the conception and design of the work and approved the final manuscript. AV, PV and $\mathrm{BL}$ contributed to acquisition and interpretation of data for the work. $\mathrm{CB}, \mathrm{AV}$ and $\mathrm{BL}$ contributed to Data analysis. $\mathrm{CB}$ and $\mathrm{AV}$ contributed to drafting the work, all authors contributed to revising it critically for important intellectual content.

\section{References}

1. von Elm E, Osterwalder JJ, Graber C, Schoettker P, Stocker R, Zangger P, et al. Severe traumatic brain injury in Switzerland - feasibility and first results of a cohort study. Swiss Med Wkly. 2008; 138: 327-334.

2. Attaques cérébrales. OBSAN. 2021.

3. Statistique des accidents. 2021.

4. Freytes IM, Sullivan M, Schmitzberger M, LeLaurin J, Orozco T, EliazarMacke $\mathrm{N}$, et al. Types of stroke-related deficits and their impact on family caregiver's depressive symptoms, burden, and quality of life. Disabil Health J. $2021 ; 14: 101019$.

5. Polinder S, Haagsma JA, van Klaveren D, Steyerberg EW, van Beeck EF. Health-related quality of life after TBI: a systematic review of study design, instruments, measurement properties, and outcome. Popul Health Metr. 2015; 13.

6. Carney N, Totten AM, O'Reilly C, Ullman JS, Hawryluk GWJ, Bell MJ, et al. Guidelines for the Management of Severe Traumatic Brain Injury. Neurosurgery. 2017; 80: 6-15. 
7. Michel $\mathrm{P}$, Arnold M, Hungerbühler H-J, Müller F, Staedler C, Baumgartne $\mathrm{RW}$, et al. Decompressive craniectomy for space occupying hemispheric and cerebellar ischemic strokes: Swiss recommendations. Int J Stroke. 2009; 4 : 218-223.

8. Brainin M, Barnes M, Baron J-C, Gilhus NE, Hughes R, Selmaj K, Guideline Standards Subcommittee of the EFNS Scientific Committee. Guidance for the preparation of neurological management guidelines by EFNS scientific task forces--revised recommendations. Eur J Neurol. 2004; 11: 577-581.

9. Keith RA, Granger CV, Hamilton BB, Sherwin FS. The functional independence measure: a new tool for rehabilitation. Adv Clin Rehabil. 1987; 1: 6-18.

10. von Steinbüchel N, Wilson L, Gibbons H, Hawthorne G, Höfer S, Schmid $\mathrm{S}$, et al. Quality of Life after Brain Injury (QOLIBRI): scale development and metric properties. J Neurotrauma. 2010; 27: 1167-1185.

11. Wilson L, Marsden-Loftus I, Koskinen S, Bakx W, Bullinger M, Formisano R, et al. Interpreting Quality of Life after Brain Injury Scores: Cross-Walk with the Short Form-36. J Neurotrauma. 2017; 34: 59-65.
12. Cooper DJ, Rosenfeld JV, Murray L, Arabi YM, Davies AR, D'Urso P, et al. Decompressive Craniectomy in Diffuse Traumatic Brain Injury. 2011; 364: 1493-1502.

13. Hutchinson PJ, Kolias AG, Timofeev IS, Corteen EA, Czosnyka M, Timothy $\mathrm{J}$, et al. Trial of Decompressive Craniectomy for Traumatic Intracranial Hypertension. N Engl J Med. 2016; 375: 1119-1130.

14. Rauen K, Reichelt L, Probst $P$, Schäpers B, Müller $F$, Jahn K, et al. Decompressive Craniectomy Is Associated With Good Quality of Life Up to 10 Years After Rehabilitation From Traumatic Brain Injury. Crit Care Med. 2020; 48: 1157-1164.

15. van Velzen JM, van Bennekom CAM, Edelaar MJA, Sluiter JK, FringsDresen MHW. How many people return to work after acquired brain injury? a systematic review. Brain Inj. 2009; 23: 473-488. 